separately, and pooled the results. The findings are expressed as odds ratios (OR) with 95\% confidence intervals $(95 \% \mathrm{Cl})$.

Results: We identified an elevated risk for psychiatric $(\mathrm{OR}=1.34,95 \% \mathrm{Cl}=1$; $1.78)$ and for affective disorders $(\mathrm{OR}=2.19,95 \% \mathrm{Cl}=1.17 ; 4.1)$ in people hospitalized for rheumatic diseases. We did not find a statistically significant association with organic, psychotic and anxiety disorders.

Conclusion: There is an increased risk for experiencing a psychiatric disorder in the period of 3 years after a rheuma-related hospitalization.

References:

[1] Shen C-C, Hu L-Y, Yang AC, Kuo BI-T, Chiang Y-Y, Tsai S-J. Risk of Psychiatric Disorders following Ankylosing Spondylitis: A Nationwide Population-based Retrospective Cohort Study. The Journal of Rheumatology. 2016;43(3).

[2] Park J-S, Jang H-D, Hong J-Y, Park Y-S, Han K, Suh S-W, et al. Impact of ankylosing spondylitis on depression: a nationwide cohort study. Scientific Reports. 2019;9(1):6736.

[3] Hsu C-C, Chen S-C, Liu C-J, Lu T, Shen C-C, Hu Y-W, et al. Rheumatoid Arthritis and the Risk of Bipolar Disorder: A Nationwide Population-Based Study. PLOS ONE. 2014;9(9).

[4] Sundquist K, Li X, Hemminki K, Sundquist J. Subsequent Risk of Hospitalization for Neuropsychiatric Disorders in Patients With Rheumatic Diseases: A Nationwide Study From Sweden. Archives of General Psychiatry. 2008;65(5):501-7.

Acknowledgments: Supported by the project (Ministry of Health Czech Republic) for conceptual development of research organization 00023728 (Institute of Rheumatology).

Disclosure of Interests: Tomáš Formánek: None declared, Karolina Mladá: None declared, Marketa Husakova Speakers bureau: Novartis

DOI: 10.1136/annrheumdis-2020-eular.3719

\section{SAT0590 ORAL MICROBIOTA IDENTIFIES PATIENTS WITH EARLY RHEUMATOID ARTHRITIS}

A. Esberg ${ }^{1}$, L. Johansson ${ }^{2}$, I. Johansson ${ }^{1}$, S. Rantapää Dahlqvist ${ }^{2} .{ }^{1}$ Odontology, Umeå, Sweden; ${ }^{2}$ Public Health and Clinical Medicine/Rheumatology, Umeå, Sweden

Background: Several studies have suggested a link between the two chronic inflammatory diseases, rheumatoid arthritis (RA) and periodontitis (PD) [1]. The diseases share similar environmental and genetic risks factors, e.g., smoking [2] and the HLA-DRB1 alleles [3]. Several serum markers used in the diagnosis of RA have also been found to be elevated in PD, e.g., anti-citrullinated proteins antibodies (ACPA) and rheumatoid factor (RF) [4]. The connection between PD and RA has been suggested to be explained by several periodontal pathogens, e.g., Aggregatibacter actinomycetemcomitans and Porphyromonas gingivalis, which have been suggested to induce the production of autoantibodies $[5,6]$. Objectives: To investigate the composition of the concerted saliva microbiota and its role in the development of RA, with the aim of improving the diagnostic tools. Methods: $16 \mathrm{~S}$ ribosomal RNA gene sequencing of saliva bacterial DNA isolated from a total of 61 early RA (eRA) patients and 59 healthy controls was made. The eRA (symptoms $\leq 12$ months) was diagnosed at an Early Arthritis Clinic (fulfilling the 1987 ARA criteria) and matched with the controls for sex and age, except for two of the elderly cases. None of the individuals included in the study had taken antibiotics during the preceding 3 months. No one of the cases were treated with anti-rheumatic drugs except for corticosteroids in 16 cases the latest month. Results: All participants were classified into three hierarchical cluster groups based on their saliva microbiota and the distribution of eRA cases versus controls differed distinctly between the cluster groups. The microbiota from the eRA had higher species richness, differed in beta-diversity, and was enriched for species in the Fusobacterium and Porphyromonas genera, and for the Alloprevotella tannerae, Campylobacter gracilis, Capnocytophaga leadbetteri, Filifactor alocis, Fusobacterium nucleatum subsp. polymorphum, Neisseria elongate, Porphyromionas endodontalis and Prevotella pleuritidis species compared to controls. Combining two topped ranked species, $A$. tannerae and Catonella morbi significantly predicted eRA with an AUC score of 0.86 and a specificity and sensitivity of 0.80 and 0.85 , respectively. The predicted functions of the microbiota in eRA patients were dominated by fatty acid metabolism, ornithine metabolism, glucosylceramidase, sphingolipids, beta-lactamase resistance, biphenyl degradation and 17-beta-estradiol 17-dehydrogenase metabolism.

Conclusion: In this study a difference in oral microbiota diversity between eRA patients and healthy controls could be shown. Some of the eRA-associated oral bacteria have previously been suggested to play an aetiological role in the development of RA, but others have not been recognized earlier, such as A. tannerae, F. alocis, F. nucleatum subsp. polymorphum, and P. endodontalis, and may therefore be useful in RA risk assessment.

References:

[1] Fuggle, N.R., et al., Hand to Mouth:A Systematic Review and Meta-Analysis of the Association between Rheumatoid Arthritis and Periodontitis. Front Immunol, 2016. 7: p. 80.
[2] Heliovaara, M., et al., Smoking and risk of rheumatoid arthritis. J Rheumatol, 1993. 20(11): p. 1830-5.

[3] Katz, J., et al., Human leukocyte antigen (HLA) DR4. Positive association with rapidly progressing periodontitis. J Periodontol, 1987. 58(9): p. 607-10.

[4] Mikuls, T.R., et al., Periodontitis and Porphyromonas gingivalis in patients with rheumatoid arthritis. Arthritis Rheumatol, 2014. 66(5): p. 1090-100.

[5] Konig, M.F., et al., Aggregatibacter actinomycetemcomitans-induced hypercitrullination links periodontal infection to autoimmunity in rheumatoid arthritis. Sci Transl Med, 2016. 8(369): p. 369ra176.

[6] Rosenstein, E.D., et al., Hypothesis: the humoral immune response to oral bacteria provides a stimulus for the development of rheumatoid arthritis. Inflammation, 2004. 28(6): p. 311-8.

Disclosure of Interests: None declared

DOI: 10.1136/annrheumdis-2020-eular.2416

\section{SAT0591 REAL-WORLD PATTERNS OF PEGLOTICASE USE FOR TREATMENT OF GOUT IN THE U.S.}

S. Chen ${ }^{1}$, J. Liu', S. Kim ${ }^{1} .{ }^{1}$ Brigham and Women's Hospital, Boston, United States of America

Background: Pegloticase is used in severe refractory gout or in cases of intolerance to other urate lowering therapies but the real-world use of pegloticase since its U.S. Food and Drug Administration approval in 2010 is not known.

Objectives: We sought to evaluate the real-world patterns of pegloticase use in the U.S. and the incidence of safety outcomes with its use.

Methods: We conducted a retrospective descriptive study using data from two U.S. commercial insurance claims databases (MarketScan and Optum, 2010 2018). We identified new initiators of pegloticase with $\geq 1$ gout diagnosis code in the 365-day baseline period prior to pegloticase initiation. We measured the number and duration of pegloticase therapy. We assessed the risk of anaphylaxis cardiovascular events including myocardial infarction or stroke, hospitalization for heart failure (new onset or exacerbations) while receiving pegloticase therapy.

Results: Among 2.9 million patients with $\geq 1$ diagnosis code for gout, we identified only 483 (179 in Optum and 304 in MarketScan) pegloticase initiators. The mean age and \% female was 55.6 years, $10.9 \%$ for MarketScan and 60.6 years and $17.3 \%$ for Optum (Table). Cardiovascular comorbidities were prevalent in both cohorts. Hypertension was present in up to $85 \%$, diabetes mellitus in $38 \%$, chronic kidney disease in $46 \%$, and heart failure in $21 \%$ of the patients. As expected, use of gout-related medications at baseline was common. The median duration of pegloticase therapy was 93 days (interquartile range [IQR] 56-186) in MarketScan and 105 (IQR 56-127) in Optum. The median number of pegloticase infusions was 4 (IQR 2-10) for MarketScan and 5 (IQR 2-12) in Optum. In MarketScan, 57 (18.8\%) patients switched to allopurinol, $64(21.1 \%)$ to febuxostat, and $2(0.7 \%)$ to probenecid during the mean 0.5-year followup time. Similarly, during the mean 0.5-year followup time in Optum, 38 (21.2\%) patients switched to allopurinol, 34 (19.0\%) to febuxostat, and $2(1.1 \%)$ to probenecid. During the mean 0.5-year follow-up time on pegloticase, there were $3(0.6 \%)$ anaphylaxis, $7(1.4 \%)$ composite cardiovascular $31(6.4 \%)$ heart failure hospitalizations, and $3(0.6 \%)$ deaths in both datasets.

Conclusion: Pegloticase is rarely used in gout, and the median duration of pegloticase therapy was 3 months. There were few anaphylaxis events captured in this claims-b ased study, while heart failure hospitalizations were common.

Table. Select baseline characteristics of pegloticase initiators

\begin{tabular}{lll}
\hline & MarketScan & Optum \\
\hline $\mathrm{N}$ & 304 & 179 \\
Mean age (SD), years & $55.62(12.83)$ & $60.58(12.85)$ \\
Female sex, \% & 10.9 & 17.3 \\
Comorbidities & & \\
Hypertension, \% & 73.0 & 84.9 \\
Diabetes mellitus, \% & 34.9 & 38.0 \\
Heart failure, \% & 12.5 & 20.7 \\
Coronary artery disease, \% & 12.8 & 26.3 \\
Chronic kidney disease, \% & 34.2 & 45.8 \\
Malignancy, \% & 10.2 & 8.4 \\
Medication use & & 40.0 \\
NSAID/COXIB use, \% & 51.3 & 65.4 \\
Colchicine use, \% & 72.0 & 47.5 \\
Allopurinol use, \% & 33.6 & 36.9 \\
Febuxostat use, \% & 38.8 & 4.5 \\
Probenecid use, \% & 8.2 & 72.1 \\
Oral steroid use, \% & 67.1 & 6.7 \\
Anakinra use, \% & 5.9 &
\end{tabular}

Disclosure of Interests: Sarah Chen Employee of: After finishing the work for this abstract, she has moved to work for Gilead., Jun Liu: None declared, Seoyoung Kim Grant/research support from: Research grants from Pfizer, AbbVie, Bristol-Myers Squibb and Roche for unrelated studies

DOI: 10.1136/annrheumdis-2020-eular.1253 